journal club

\title{
Verbesserung und Update der PML-Risikoberechnung
}

Fragestellung: Mitarbeiter des Herstellers von Natalizumab stellen das Update ihres Algorithmus für die Einschätzung des PML-Risikos im Verlauf der Therapie mit Natalizumab vor.

Hintergrund: Die Behandlung mit dem bei der schubförmigen Multiplen Sklerose (MS) eingesetzten Medikament Natalizumab kann zur Entwicklung einer JC-Virus(JCV-)vermittelten progressiven multifokalen Leukenzephalopathie (PML) führen. Bekannte Risikofaktoren für die PML sind die vorherige Behandlung mit immunsupprimierenden Medikamenten, die Langzeitbehandlung mit Natalizumab sowie der Nachweis von Anti-JCV-Antikörpern im Serum über bestimmten Schwellenwerten. Um die Behandlung mit Natalizumab möglichst sicher durchzuführen, informiert der Hersteller seit dem Aufkommen der ersten PML-Fälle regelmäßig über die Berechnung des PMLRisikos für Patienten unter Berücksichtigung der oben

Ho PR, Koendgen $\mathrm{H}$, Campbell $\mathrm{N}$ et al. Risk of natalizumabassociated progressive multifocal leukoencephalopathy in patients with multiple sclerosis: a retrospective analysis of data from four clinical studies. Lancet Neurol 2017; 16: $925-33$ genannten Risikofaktoren. Basis für diese Berechnungen war lange Zeit die Arbeit von Bloomgren et al. (Biogen) von 2012, die aber zum Teil auf Schätzungen basierte und wiederholt wegen der zugrunde liegenden Arithmetik ohne Einbezug der Therapieabbrecher sowie der Darstellung des Risikos in Intervallen kritisiert wurde. Da die EMA (Europäische Arzneimittel-Agentur) ebenfalls 2015/2016 eine Neuberechnung forderte, publizierte der Hersteller mit dieser Arbeit nun die verbesserten Algorithmen.

Patienten und Methodik: Auswertung von vier klinischen Beobachtungsstudien (STRATIFY-II, STRATA, TOP und TYGRIS) mit insgesamt 37.249 mit Natalizumab behandelten Patienten, in deren Verlauf 156 PML-Fälle berichtet wurden.

Ergebnisse: Das PML-Risiko variiert je nach Kombination der Risikofaktoren zwischen 0,07 pro 1.000 behandelte Patienten (im Fall eines negativen JCV-Antikörperstatus) und 2,7\% bei positivem JCV-Antikörperstatus, sechs Jahren Therapiedauer sowie vorheriger Immunsuppression. Verallgemeinernd kann man sagen, dass ein positiver Anti-JCV-Serostatus (Indexwert 0,9-1,5) das Risiko im Vergleich zu einem Langzeitpatienten mit niedrigem Index $(<0,9)$ um das fünffache erhöht, ein Indexwert $>1,5$ um das 18-fache und vorherige Immunsuppression um das 17-fache. Die genauen Risiken für die jeweiligen Kombinationen der verschiedenen Faktoren finden sich im Manuskript als Tabellen.

Schlussfolgerung: Die Analysen bestätigen grundsätzlich die Validität der drei bekannten Risikofaktoren.

\section{- Kommentar von Heinz Wiendl, Münster}

\section{Überflüssige Aktualisierung der Zahlen zur PML-Risikoberechnung}

Dieses Update liefert einen fundierten Überblick über das PMLRisiko im Zusammenhang mit Natalizumab. Die Neuheit des Manuskripts sind die gezeigten Kaplan-Meier-Kurven für die Darstellung des kumulativen PML-Risikos. Diese waren von der EMA im Rahmen des Artikel-20-Verfahrens gefordert worden und der Hersteller bestätigt hier empirisch das bereits Anfang 2017 hypothetisierte Höchstrisiko von 1:37 bei immunsupprimierten Anti-JCV-seropositiven Langzeitpatienten. Der Datensatz erlaubt mit oben genannten Einschränkungen die Abschätzung des PML-Risikos von Patienten während ihrer Therapie mit Natalizumab. Der behandelnde Arzt kann anhand des jeweiligen Krankheitsprofils des Patienten entscheiden, inwieweit das PMLRisiko gerechtfertigt ist. Während das Risiko von JCV-seronegativen Patienten mit 1:15.000 nach wie vor bei halbjährlicher Kontrolle des JCV-Serostatus als sehr gering einzuschätzen ist, ist das kumulative Risiko von 1:37 eines Hochrisikopatienten nur schwer zu rechtfertigen.

Natalizumab hat wegen seiner hohen Wirksamkeit nach wie vor einen Platz im Behandlungsspektrum der MS. Problematisch bleibt allerdings, dass die Inzidenz von PML-Fällen insge- samt trotz Implementierung von Risikominimierungsstrategien weiter steigt. Obwohl diese Arbeit die Rolle des Anti-JCV-Antikörperindex statistisch robust als Risikobiomarker unterstützt, bleibt der Anti-JCV-Antikörperstatus insgesamt leider ein zwar sensitiver, aber nicht sehr spezifischer Marker für ein PML-Risiko. Für Biomarker außerhalb des JCV-Serostatus besteht weiterhin hoher Bedarf. Insgesamt ist diese wichtige Arbeit als Korrektur und Update des PML-Risikoalgorithmus zu verstehen, aber nicht als echte Neuerung.

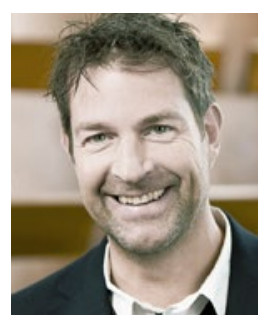

Univ.-Prof. Dr. med. Heinz Wiendl, Münster

Direktor der Klinik für Allgemeine Neurologie, Universitätsklinikum Münster

E-Mail: heinz.wiendl@ukmuenster.de 